\title{
A NOTE ON NONPARAMETRIC IDENTIFICATION OF DISTRIBUTIONS OF RANDOM COEFFICIENTS IN MULTINOMIAL CHOICE MODELS
}

\author{
Jeremy T. Fox \\ Working Paper 23621 \\ http://www.nber.org/papers/w23621 \\ NATIONAL BUREAU OF ECONOMIC RESEARCH \\ 1050 Massachusetts Avenue \\ Cambridge, MA 02138 \\ July 2017
}

The views expressed herein are those of the author and do not necessarily reflect the views of the National Bureau of Economic Research.

NBER working papers are circulated for discussion and comment purposes. They have not been peer-reviewed or been subject to the review by the NBER Board of Directors that accompanies official NBER publications.

(C) 2017 by Jeremy T. Fox. All rights reserved. Short sections of text, not to exceed two paragraphs, may be quoted without explicit permission provided that full credit, including $($ ) notice, is given to the source. 
A Note on Nonparametric Identification of Distributions of Random Coefficients in Multinomial Choice Models

Jeremy T. Fox

NBER Working Paper No. 23621

July 2017

JEL No. C25,L0

\begin{abstract}
I prove that the joint distribution of random coefficients and additive errors is identified in a mulltinomial choice model. No restrictions are imposed on the support of the random coefficients and additive errors. The proof uses large support variation in choice-specific explanatory variables following Lewbel (2000) but does not rely on an identification at infinity technique where the payoffs of all but two choices are set to minus infinity.
\end{abstract}

Jeremy T. Fox

Rice University

Department of Economics - MS22

Baker Hall

P.O. Box 1892

Houston, TX 77251-1892

and NBER

jeremyfox@gmail.com 


\title{
A Note on Nonparametric Identification of Distributions of Random Coefficients in Multinomial Choice Models
}

\author{
Jeremy T. Fox \\ Rice University and NBER
}

July 2017

Two key papers on identifying the distribution of random coefficients and additive errors in binary (two) choice have not been extended to multinomial (two or more, importantly three or more) choice (Ichimura and Thompson, 1998; Gautier and Kitamura, 2013). Under large support on choice-specific explanatory variables, one can always turn a multinomial choice model with three or more goods into a binary choice model by setting the explanatory variables for all but two goods to be minus infinity. This identification at infinity approach does not identify the joint distribution of all unobservables in the model if there are unobservables that enter only the utilities of certain goods. Establishing the identification of the joint distribution of random coefficients and additive unobservables in the multinomial choice model is the point of this note.

The argument in this note might be considered an extension to multinomial choice of the brief argument about identifying a distribution of random coefficients in binary choice in Lewbel (2014, Section 8). Our identification proof uses the results on identifying distributions of random coefficients in seemingly unrelated regressions by Masten (forthcoming). A key step of our identification proof is also found in Berry and Haile (2010) and Fox and Gandhi (2016).

There are some related papers on multinomial choice. Lewbel (2000) considers multinomial choice in a semiparametric setting but does not explicitly identify a distribution of random coefficients. Fox and Gandhi (2016) study the topic of this note: nonparametric identification in multinomial choice models, where the example of a linear-in-random-coefficients model is a special case of their analysis. However, Fox and Gandhi assume that the distribution of random coefficients and additive errors takes on unknown finite support in the appropriate real space. This note avoids the unknown finite support assumption. Fox, Kim, Ryan and Bajari (2012) nonparametrically identify a distribution of random coefficients on continuous explanatory variables but rely on the additive, good-specific, unobservables having a known distribution such as the type I extreme value or logit distribution. In this note, the joint distribution of the good-specific, additive unobservables is identified.

Consider a multinomial choice model with random coefficients. Let $i$ index a consumer. There are $J$ inside goods and one outside good, called choice 0 . The outside good has a utility normalized to $u_{i, 0}=0$. The inside 
goods have utilities

$$
u_{i, j}=\beta_{i}^{\prime} x_{i, j}+\epsilon_{i, j}
$$

where $x_{i, j}$ is a vector of observables for choice $j$ and consumer $i, \beta_{i}$ is consumer $i$ 's vector of random coefficients on the explanatory variables, and $\epsilon_{i, j}$ is an additive unobservable for choice $j$ and consumer $i$. We do not impose that $\epsilon_{i, j}$ has mean zero and we do not allow intercept terms in $x_{i, j}$. Note that $\beta_{i}$ is common to all choices.

We impose the scale normalization that one element of the vector $\beta_{i}$ has the value \pm 1 for each $i$. This rules out this coefficient can be zero. Let $w_{i, j}$ be the corresponding scalar element of $x_{i, j}$ and let $\tilde{x}_{i, j}$ be all other elements of $x_{i, j}$, so that $x_{i, j}=\left(w_{i, j}, \tilde{x}_{i, j}\right)$. Then the utility to choice $j$ can be rewritten as

$$
u_{i, j}= \pm 1 w_{i, j}+\tilde{\beta}_{i}^{\prime} \tilde{x}_{i, j}+\epsilon_{i, j}
$$

where $\tilde{\beta}_{i}$ corresponds to the random coefficients on only the items in $\tilde{x}_{i, j}$. We further impose that the coefficient on $w_{i, j}$ is either +1 for all consumers $i$ or is -1 for all consumers $i$.

We consider i.i.d. observations on $\left(y_{i}, x_{i}\right)$, where $y_{i}$ is the choice that maximizes $u_{i, j}$ over $\{0,1, \ldots, J\}$. Given this, we can nonparametrically identify conditional choice probabilities $\operatorname{Pr}\left(y_{i}=j \mid x_{i}\right)$. We assume that $x_{i}$ is independent of $\gamma_{i}$. We can discuss endogeneity with various methods in the literature such as Lewbel (2000). We leave that for extensions.

The sign of the coefficient on $w_{i, j}$ is learned in identification; a positive coefficient on $w_{i, j}$ corresponds to higher values of $w_{i, j}$ increasing the choice probability of good $j$, other explanatory variables held constant. We make this formal in the proof of identification.

Let $x_{i}=\left(x_{i, 1}, \ldots, x_{i, J}\right), w_{i}=\left(w_{i, 1}, \ldots, w_{i, J}\right), \tilde{x}_{i}=\left(\tilde{x}_{i, 1}, \ldots, \tilde{x}_{i, J}\right)$ and $\epsilon_{i}=\left(\epsilon_{i, 1}, \ldots, \epsilon_{1, J}\right)$ all be thought of as long vectors. Also, define $\gamma_{i}=\left(\beta_{i}, \epsilon_{i}\right)$ and think of it as another long vector. Note that $\gamma_{i}$ is a heterogeneous parameter vector, not a homogeneous parameter. In what follows, we drop the $i$ subscript.

The only unknown object in this model is $F(\gamma)$, the joint distribution of the additive unobservables $\epsilon$ and the random coefficients $\beta$. Because we will not restrict the support of each $\epsilon_{j}$ and $\beta$, a sufficient condition for identification of $F$, as shown in this note, will be that the support of $x$, reordered as $x=(w, \tilde{x})$ is $\mathbb{R}^{\operatorname{dim}(w)} \times \tilde{X}$, where the support of $w$ is the large support $\mathbb{R}^{\operatorname{dim}(w)}$ and the support of the vector $\tilde{x}$ is $\tilde{X}$, a closed superset of an open subset of the real space $\mathbb{R}^{\operatorname{dim}(\tilde{x})}$. $^{1}$ Having support on the product space $\mathbb{R}^{\operatorname{dim}(w)}$ for $w$ and a superset of an open subset of $\mathbb{R}^{\operatorname{dim}(\tilde{x})}$ for $\tilde{x}$ rules out the entire vector $x$ containing polynomial terms of other elements in $x$, interactions of two elements also in $x$, or the same element of $x$ entering the utility of different goods. Continuous support on all elements in $x$ rules out discrete $x$. Large support is needed for $w$ but not for $\tilde{x}$.

Some version of a large support condition, while not particularly attractive, is necessary to achieve identification. Consider the special case of our model where there is one inside good $(J=1)$, one outside good and no $\tilde{x}$. This is binary choice, which has been extensively studied in the literature. Then the utility of good 1 is

$$
u_{1}= \pm 1 w_{1}+\epsilon_{1}
$$

\footnotetext{
${ }^{1}$ Often the term mathematical term support is defined to be a closed set.
} 
and the utility of good 0 is still $u_{0}=0$. We can identify the sign of the common coefficient on $w_{1}$ by seeing whether $\operatorname{Pr}\left(y=1 \mid w_{1}\right)$ is monotonically increasing or decreasing in the scalar $w_{1}$. Let $\bar{w}_{1}=+w_{1}$ or $\bar{w}_{1}=-w_{1}$, as appropriate. In this example, $\gamma=\epsilon_{1}$. We can identify the distribution $F_{\epsilon}\left(\epsilon_{1}\right)$ at the point of evaluation $\epsilon_{1}^{\star}$ as follows

$$
F_{\epsilon}\left(\epsilon_{1}^{\star}\right)=\operatorname{Pr}\left(\epsilon_{1} \leq \epsilon_{1}^{\star}\right)=\operatorname{Pr}\left(\epsilon_{1} \leq-\bar{w}_{1} \mid-\bar{w}_{1}=\epsilon_{1}^{\star}\right)=\operatorname{Pr}\left(y=0 \mid-\bar{w}_{1}=\epsilon_{1}^{\star}\right) .
$$

If $\epsilon$ takes on support on $\mathbb{R}$, then this argument shows that $\bar{w}_{1}$ must also take on support on all of $\mathbb{R}$ to identify $F_{\epsilon}\left(\epsilon_{1}\right)$ over its entire support. As $\operatorname{Pr}\left(y=0 \mid \bar{w}_{1}\right)+\operatorname{Pr}\left(y=1 \mid \bar{w}_{1}\right)=1$, all available data on conditional choice probabilities is used in the identification argument. This binary choice example is a special case of our model so it shows that some large support is necessary for identification.

We are now ready to state the main identification theorem.

Theorem 1. If the support of $x$ is as stated previously, $\gamma$ is independent of $x, \gamma$ has finite absolute moments, and the distribution of $\gamma$ is uniquely determined by its moments, then $F(\gamma)$ is identified.

Proof. The argument works in three steps. First, we identify the sign of the coefficient on the explanatory variables $w_{j}$ that form the scale normalization. Second, we use $w$ to trace out the CDF of utility values (other than from $w$ ) conditional on the other explanatory variables in $\tilde{x}$. Third, we cite work by Masten (forthcoming, Theorem 1) on seemingly unrelated regressions with random coefficients to identify the distribution of the random coefficients and additive unobservables.

First, we identify the sign of the coefficient on each $w_{j}$, which has been normalized to \pm 1 . We observe conditional choice probabilities $\operatorname{Pr}(y=j \mid x)$ and the vector $w$ is a subvector of $x$. If $\operatorname{Pr}(y=j \mid x)$ is monotonically increasing in $w_{j}$ for some $j$, then the coefficient on each $w_{j}$ is +1 . If it is decreasing, then the coefficient on each $w_{j}$ is -1 . Let $\bar{w}_{j}=+w_{j}$ or $\bar{w}_{j}=-w_{j}$, as appropriate based on the sign of the coefficient on $w_{j}$. Let $\bar{w}=\left(\bar{w}_{1}, \ldots, \bar{w}_{J}\right)$.

Second, we use arguments motivated by Lewbel (2000) to trace a CDF of utility values (other than from $w$ ) conditional on the explanatory variables in $\tilde{x}$. Define

$$
\tilde{u}_{j}=\tilde{\beta}^{\prime} \tilde{x}_{j}+\epsilon_{j}
$$

and $\tilde{u}=\left(\tilde{u}_{1}, \ldots, \tilde{u}_{J}\right)$. Let the CDF of the vector $\tilde{u}$ conditional on the vector $\tilde{x}$ be $G_{\tilde{u}}(\tilde{u} \mid \tilde{x})$. Let $\tilde{u}^{\star}$ be a point of evaluation of the CDF. Then, for arbitrary $\tilde{x}$,

$$
G_{\tilde{u}}\left(\tilde{u}^{\star} \mid \tilde{x}\right)=\operatorname{Pr}\left(\tilde{u} \leq \tilde{u}^{\star} \mid \tilde{x}\right)=\operatorname{Pr}\left(\tilde{u} \leq-\bar{w} \mid-\bar{w}=\tilde{u}^{\star}, \tilde{x}\right)=\operatorname{Pr}\left(y=0 \mid-\bar{w}=\tilde{u}^{\star}, \tilde{x}\right) .
$$

All the lower case letters in the above display equation are vectors, except for $y$. This argument identifies the $\operatorname{CDF} G_{\tilde{u}}\left(\tilde{u}^{\star} \mid \tilde{x}\right)$ at all points of evaluation because $w$ has full support on $\mathbb{R}^{\operatorname{dim}(w)}$. This argument is not new. It appears in Berry and Haile (2010) and Fox and Gandhi (2016).

Third, we use Masten (forthcoming, Theorem 1) to identify the distribution of $\gamma$ itself. From the previous step we observe $G_{\tilde{u}}(\tilde{u} \mid \tilde{x})$ for $\tilde{x}$ varying in an open set, which is equivalent to the distribution that Masten 
(forthcoming, Theorem 1) maintains is observed in a seemingly unrelated regression model. ${ }^{2}$ Therefore, $F(\gamma)$ is identified for any $J$.

This identification argument relies heavily on large support of $w$, meaning $w$ has support $\mathbb{R}^{\operatorname{dim}(w)}$. The argument does not use identification at infinity. In multinomial choice, we can precisely define identification at infinity to mean a step of an identification proof that sets $w_{j}=-\infty$ for $J-1$ inside goods and uses an analysis from binary choice on the resulting pair of an inside good and an outside good. One can inspect the identification proof to see that this type of argument is not used. Indeed, the second step of the proof is explicitly incompatible with identification at infinity, as the vector $w$ is used over its full support $\mathbb{R}^{\operatorname{dim}(w)}$.

\section{References}

Berry, Steven T. and Philip A. Haile, "Nonparametric Identification of Multinomial Choice Demand Models with Heterogeneous Consumers," 2010. Yale University working paper.

Fox, Jeremy T. and Amit Gandhi, "Nonparametric Identification and Estimation of Random Coefficients in Multinomial Choice Models," RAND Journal of Economics, 2016, 47 (1), 118-139.

_ , Kyoo il Kim, Stephen Ryan, and Patrick Bajari, "The Random Coefficients Logit Model is Identified," Journal of Econometrics, 2012, 1662 (2), 204-212.

Gautier, E. and Y. Kitamura, "Nonparametric Estimation in Random Coefficients Binary Choice Models," Econometrica, 2013, 81 (2), 581-607.

Ichimura, H. and TS Thompson, "Maximum likelihood estimation of a binary choice model with random coefficients of unknown distribution," Journal of Econometrics, 1998, 86 (2), 269-295.

Lewbel, Arthur, "Semiparametric Qualitative Response Model Estimation with Unknown Heteroscedasticity or Instrumental Variables," Journal of Econometrics, 2000, 97 (1), 145-177.

_ , "An Overview of the Special Regressor Method," in "Oxford Handbook of Applied Nonparametric and Semiparametric Econometrics and Statistics," Oxford University Press, 2014, pp. 38-62.

Masten, Matthew, "Random coefficients on endogenous variables in simultaneous equations models," The Review of Economic Studies, forthcoming.

\footnotetext{
${ }^{2}$ Masten (forthcoming, Theorem 1) is stated for a seemingly unrelated regression model with two equations but this was just an editorial decision on his part and the argument applies to any number of equations. Masten (forthcoming, Theorem 1) directly applies to a model with a good-specific vector of random coefficients $\beta_{j}$ for each inside good $j$, in addition to the good-specific additive error $\epsilon_{j}$. The typical restriction is used in this paper: $\beta_{j_{1}}=\beta_{j_{2}}$ for all inside goods $j_{1}, j_{2}$. This is a special case of Masten (forthcoming, Theorem 1).
} 